\title{
Accessory Regulatory Proteins of HIV-1 and Host Restriction Factors Interactions
}

\author{
Lopez Hernandez MA ${ }^{1 *}$ and Lopez De Lucio $S^{2}$ \\ ${ }^{1}$ HIV Clinic, General Regional Hospital Na1, Mexico \\ ${ }^{2}$ Cerrada de Yalai Mz2 L2 C 148, Palmas 2, Ixtapaluca, Mexico
}

Corresponding author: Lopez Hernandez MA, HIV Clinic, General Regional Hospital №1, Mexico

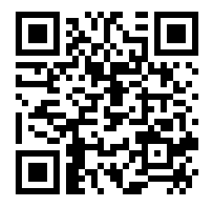

ARTICLE INFO
Received: 仹 October 21, 2020
Published: October 30, 2020

Citation: Lopez Hernandez MA, Lopez De Lucio S. Accessory Regulatory Proteins of HIV-1 and Host Restriction Factors Interactions. Biomed J Sci \& Tech Res 31(4)-2020. BJSTR. MS.ID.005120.

Keywords: HIV-1 Accessory Proteins; Restriction Factors; APOBEG3G; BST2; SERINC5; SERINC3; Vif; Vpu; Nef; Vpr

\section{ABSTRACT}

Primate immunodeficiency viruses, including HIV-1, The presence of accessory genes are characteristic of them, such as vif, vpr, vpx, vpu, and nef. The current knowledge indicates that none of this lentiviral accessory proteins has enzymatic activity. These proteins interact with cellular ligands to either act as adaptation of molecules to redirect the normal function of host factors for virus-specific purposes or to inhibit a normal host function by mediating degradation or causing intracellular sequestration of the factors involved. The intrinsic antiviral factors that restrict viral replication in host cells are countered by accessory proteins the HIV-1 and other lentiviruses had acquired along their evolution.

Abbreviations: HIV-1: Human Immunodeficiency Virus Type 1; SIV: Simian Immunodeficiency Virus; IFN: Interferon; GPI: Glycophosphatidylinositol; MHC-1: Major Histocompatibility Complex Class 1; CRLs: Cullin-Ring Ligases; Vpr: Viral Protein r; VprBP: Vpr Binding Protein

\section{Mini Review}

\section{Accessory Regulatory Proteins}

When the human immunodeficiency virus type 1 was cloned and sequenced for first time, the researchers were surprised to find that this virus contained several additional open reading frames. in addition to the prototypical retroviral gag, pol, and env genes; Collectively, they were termed accessory genes due to a lack of understanding of their biological functions. Viruses need to counteract intrinsic antiviral factors that restrict viral replication in host cells and for this purpose HIV and SIV might have acquired accessory proteins along their evolution. Virus protein $\mathrm{U}$ or $\mathrm{Vpu}$ is a lentiviral viroporin of 81 aminoacid, that is specific of HIV-1.This accessory protein is a membrane phosphoprotein, oligomeric with diversified biological functions. Vpu accessory protein is implicated in CD4 degradation, involving the ubiquitin proteasome pathway as well as in the release of virions from host cells. Viral infectivity factor or Vif is a highly conserved $23 \mathrm{kDa}$ phosphoprotein, Vif is binded to the restriction factor APOBEG3AG which is cellular cytidine deaminases that provides inmmunity against HIV and promotes its degradation.
Vif is outstanding for the infectivity of HIV-1 virions that depends on the cell type. Vif is necessary to the synthesis of infectious virions in macrophages, lymphocytes, and dendritic cells expressing hA3G. Negative factor or Nef, is a membrane associated phosphoprotein with the $\mathrm{N}$-terminal myristoylated. Nef is involved in many functions during the replication cycle of the VIH-1, therefore, it is believing that plays an important role in the increase virus infectivity and in cell apoptosis. Virus protein $\mathrm{R}$ or $\mathrm{Vpr}$ is a regulatory protein with nucleocytoplasmic shuttling. Vpr through the nuclear import of the preintegration complex play an important part in replication of the virus, Vpr also appears to cause its host cells to arrest their cell cycle in the G2 phase. Vpx is an HIV-2 and SIV encoded protein which functions are associated with Vpr. The HIV-1 is strictly adapted to human host.

Cellular activation has been considered a requirement for HIV infection of CD4 T cells, as HIV fails to infect resting CD4 T cells from peripheral blood. Infection is aborted either during reverse transcription [1]. Like other primate retroviruses, the human immunodeficiency virus type 1 (HIV-1) is characterized by the 
presence of accessory genes, for example: vif, vpr, vpx, vpu, and nef. Current knowledge indicates that none of the primate lentiviral accessory proteins has enzymatic activity. Extensive studies on the underlying mechanism have revealed that Vif is one of viral accessory proteins, is critical for the HIV-1 species tropism in addition to Gag-capsid protein. Another auxiliary protein is Vpu that also has been demonstrated that affects this HIV-1 property. The restriction factors are multitaskers and highly conserved, one of the restriction factors function is the regulation of cross-species infections. Consequently, in their natural hosts. they are less effective against viruses.

\section{Vif (Viral Infectivity Factor) and APOBEC3G}

APOBEC are a family of cellular cytidine deaminases and represent aroup of proteins recently identified. APOBEC protect the cell from endogenous mobile retro elements and provide immunity against infection by retroviruses. Vif is an accessory protein of HIV-1 required for productive infectivity of T-lymphocytes, macrophages and dendritic cells expressing hA3G [2-5]. Vif plays a fundamental roll in the viral replication, is a protein of $23 \mathrm{kD}$ that inhibits the host cell protein APOBECG3G (hA3G), which is a host defense factor human. Vif binding to hA3G since the viral entry prevents the assembly with viral particles and also mediates by the proteosomal pathway elimination through ubiquitination and degradation. hA3G binds to the HIV-1 RNA genome and attenuates the viral replication during the post entry phase of the HIV-1 infection. An important mechanism of Vif involves its ability to bind to both Elongin B/C complex of the ubiquitination machinery and to the human host defense factor APOBEC3G (hA3G).

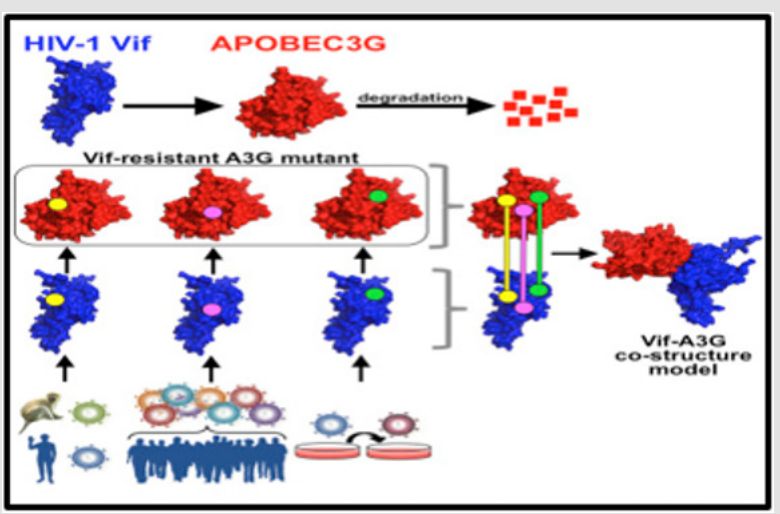

Figure 1: The human restriction factor APOBEC3G (A3G) is degraded by the HIV Vif binding. Three specific HIV Vif-A3G contact points were identified using functional assays.

Through the deamination of deoxycytidines to form deoxyuridines within single stranded DNA regions of the replicated viral genome hA3G inhibits the viral replication [6-13]. A functional Vif-A3G interface model was generated by Letko, et al. through the identification and use of specific interaction points between Vif and A3G. There are three specific HIV Vif-A3G contact points identified in this model, the A3G binding site on Vif consists of a defined, positively charged pocket using functional assays. The Vif-A3G interface is revealed by the use of protein docking Vif-A3G anchor points (Figure 1).

\section{Vpu (Viral Protein U) and BST2 (Tetherin/CD317)}

$\mathrm{Vpu}$ is an accessory viral protein that has two functions, degradation of CD4 molecule in the endoplasmic reticulum and enhancement of virion release from cells. Vpu is a type IA viroporin, encoded by human immunodeficiency virus type 1 (HIV-1), also vpu is encoded by some simian immunodeficiency virus (SIV) strains. Vpu is a small protein conformed by 81 amino acids and contain a single transmembrane domain. The functionality of Vpu is well conserved in pandemic virus isolates notwithstanding the high variability of its amino acid sequence. There is a correlation direct between structure, topology and function of Vpu pointed by a functional analysis of this membrane protein. The two biological activities of Vpu protein were charted in two separable structural domains: the transmembrane $\mathrm{N}$-terminus and the cytoplasmic C-terminus by early studies [14-16]. The CD4 receptor in infected cells is downregulated by the HIV-1 Negative Regulatory Factor (Nef) and Vpu proteins. While Vpu targets the newly synthesized CD4 molecules in the endoplasmic reticulum, Nef degrades preexisting CD4 at the plasma membrane [17].

The cytoplasmic C-terminus domain of the Vpu protein regulate this activity. The enhancement induced by Vpu of the release of viral particles from infected cells was by first time mapped in the transmembrane region of $\mathrm{Vpu}$, which suggest that $\mathrm{Vpu}$ does not interact directly with viral proteins, but rather, via cellular factors, mediates this function. On the other hand, some other studies focused on the ion channel activity of Vpu. Thus, Vpu promote budding from the plasma membrane of infected cells was suggested by the Vpu-induced movement of monovalent cations across the internal cellular membrane and the reorganization of raft structures s creating cellular conditions for that [18-22]. The identification of the interferon (IFN)- $\alpha$-induced glycoprotein Bone Marrow Stromal Cell Antigen 2 (BST2) (tetherin/CD317) has allowed the development the model based on antiviral host restriction which inhibits the release of virus and is antagonized by Vpu.

BST2 is a type II transmembrane protein with two membrane anchors, both are joined by an ectodomain; the transmembrane domain near the N-terminus and a glycophosphatidylinositol (GPI)-linked anchor at the C terminus [23-25]. Thus, tetherin has the potential to restrict replication and spread of HIV-1 in humans. Importantly, the human ortholog of tetherin contains a deletion in its cytoplasmic domain that was already present in archaic hominins but is absent from tetherin proteins of nonhuman primates. This deletion usually confers resistance to the accessory Nef protein used by most SIVs, including the precursors of HIV-1 and HIV-2, to counteract tetherin in their respective primate hosts. Pandemic HIV-1 group M strains switched from Nef to Vpu to clear 
the tetherin barrier in the human host. The Vpu proteins of group N HIV-1 strains are usually less active than group M Vpus (M-Vpus) and also has acquired some anti-tetherin activity, but they lack other functions like the ability to degrade CD4 [26-31].

\section{Nef and SERINC3, SERINC5}

Nef (Negative Regulatory Factor) is a smal myristoylated protein of 27-35 kDa that is encoded by primate lentiviruses. These include Human Immunodeficiency Viruses (HIV-1 and HIV-2) and Simian Immunodeficiency Virus, is a small peripheral membrane protein expressed early during the viral replication cycle, before the viral enzymes and structural proteins including Env. Nef associates with membranes via N-terminal myristoylation. Nef, unlike HIV-1 $\mathrm{Vpu}$, shows activity against the factor of restriction of SERINC5. This SERINC5, antagonism occurred in parallel with other Nef activities, like trans-Golgi network targeting of Lck, cell surface receptor downregulation, and inhibition of host cell actin dynamics. Nef is associated with membranes via N-terminal myristoylation. Simian immunodeficiency viruses (SIVs) use their Nef proteins to counteract the restriction factor tetherin. However, a deletion in human tethering prevents antagonism by the Nef proteins of SIVcpz and SIVgor, which represent the ape precursors of human immunodeficiency virus type 1 (HIV-1).

To promote virus release from infected cells, pandemic HIV-1 group M strains evolved Vpu as a tetherin antagonist, whilst the Nef protein of less widespread HIV-1 group 0 strains acquired the ability to target a region adjacent to this deletion. Through manipulation of the cell machinery the HIV-1 evades the host's immune response. This process involves the use of vesicular traffic to efficiently direct cell markers such as major histocompatibility complex class 1 (MHC-1) and CD4 from the plasma membrane to the endosomes, to finally degrade in the lysosome. The SERINC proteins belong a family of transmembrane proteins that are present in all eukaryotic cells. The SERINC family is comprised of five members, from SERINC1 to SERINC5, structurally characterized as having from 10 to 11 transmembrane domains. SERINC proteins participate in the biosynthesis of sphingolipids and phosphatidylserine by incorporating serine into membrane lipids SERINC3 (serine incorporator 3) and SERINC5, and in the transport of the serine amino acid across the lipid bilayer.

SERINC3/5 are recently identified as host cell inhibitors of HIV1 particle infectivity and are counteracted by the viral pathogenesis factor Nef. SERINC5 decrease the ability of the virions to fuse with the target cells by the enlargement of the fusion pore. SERINC5 could prevent the entry of the virus prior to small pore formation by promoting structural changes in envelope glycoproteins [32]. Nef antagonize the antiviral activity of SERINC5, leading to a decrease in its incorporation in the virions, because Nef removes it from the plasma membrane and sequesters it in the endosomes for its subsequent degradation [33]. Nef induces,by using the cellular transport machinery, downregulation of SERINC3 and SERINC5 from the cell membrane, mainly using the trans-Golgi network and the endolysosomal system, in a mechanism similar to that used for CD4 downregulation [34-36].

Myristoylation requires Nef to anchor in the membrane for a posttranslational change and execute sequestration of the proteins $[37,38]$.

\section{Vpr (Viral Protein R) and VprBP (DCAF1)}

The HIV protein, Vpr is critical for efficient viral infection of target CD4+ T cells and macrophages, it is a multifunctional accessory protein. $\mathrm{Vpr}$ is a 96 amino acid $14-\mathrm{kDa}$ protein $[39,40]$, Vpr is required for virus replication in non-dividing cells such as macrophages and plays an important role in the regulation of nuclear import of the HIV-1 pre-integration complex,. Vpr is incorporated into virions and functions to transport the preintegration complex into the nucleus where the process of viral integration into the host genome is completed. Numerous investigations over the last 30 years have shown that Vpr mediates many processes that aid HIV1 infection, the evasion of the immune system, and persistence in the host. Retroviruses require entry into the nucleus to replicate, hence, it is naturally restricted to those cells that undergo mitosis. Lentiviruses such as HIV-1, however, are unique among retroviruses where they are able to infect non-dividing cells $[41,42]$.

Vpr protein shuttles between cytoplasm and nucleus once the virion enters the cell, to facilitate viral replication and, at late stages in the virus life cycle, newly synthesized Vpr is exported from the cell.

Two forms of nuclear Vpr have been observed, monomers and very large complexes, $1000 \times$ larger than a monomer. One of the outstanding activities performed by Vpr is the blockade of the cell cycle at G2 after HIV-1 infection of dividing cells [43-46]. The viral LTR promoter is more active in the phase G2 of the cell cycle. Other activities of Vpr include the transcriptional modulation of immune function and the regulation of apoptosis [47-50]. Several proteomic analyses have been undertaken in an attempt to unravel the precise mechanism of action of HIV-1 Vpr. There are changes induced by Vpr to protein modulators and mediators of signaling pathways related to glycolysis and other energy processes, involved in cell cycle, cell death and DNA repair, mitochondrial activity and redox homeostasis,. Cullin-RING ligases (CRLs) comprise a large group of modular eukaryotic E3 ubiquitin ligases. DCAF1 was originally discovered as the cellular target of the HIV-1 accessory protein viral protein $r$ (Vpr), hereafter was originally named Vpr binding protein (VprBP) [51]. The substrate receptors for CRL4 have been termed DDB1 and CUL4-associated factors (DCAFs). The number of identified and putative DCAFs is in excess of 50 members and is likely to continue growing.

\section{Conclusion}

A fundamental concept to the biology of restriction factors is that HIV-1 generally evades their potent inhibitory activities in human 
cells, thereby allowing virus replication to proceed efficiently. Viruses need to counteract intrinsic antiviral factors that restrict viral replication in host cells and for this purpose lentiviruses might have acquired accessory proteins along their evolution. The understanding of the structure, function of this accessory proteins and their mechanisms of interaction with ligands in the host cell could be important for the development of new therapies for the HIV infection.

\section{References}

1. Zack JA, Arrigo SJ, Weitsman SR, Go AS, Haislip A, et al. (1990) HIV-1 entry into quiescent primary lymphocytes: molecular analysis reveals a labile, latent viral structure. Cell 61(2): 213-222.

2. Chiu YL, Greene WC (2006) APOBEC3 cytidine deaminases: distinct antiviral actions along the retroviral life cycle. J Biol Chem 281(13): 8309-8312.

3. Chiu YL, Greene WC (2006) Multifaceted antiviral actions of APOBEC3 cytidine deaminases. Trends Immunol 27(6): 291-297.

4. Chiu YL, Soros VB, Kreisberg JF, Stopak K, Yonemoto W, et al. (2005) Cellular APOBEC3G restricts HIV-1 infection in resting CD4+ T cells. Nature 435(7038): 108-114.

5. Kreisberg JF, Yonemoto W, Greene WC (2006) Endogenous factors enhance HIV infection of tissue naive CD4 T cells by stimulating high molecular mass APOBEC3G complex formation. J Exp Med 203(4): 865870.

6. Conticello SG, Harris RS, Neuberger MS (2003) The Vif protein of HIV triggers degradation of the human antiretroviral DNA deaminase APOBEC3G. Curr Biol 13(22): 2009-2013.

7. Kobayashi M, Takaori Kondo A, Miyauchi Y, Iwai K, Uchiyama T (2005) Ubiquitination of APOBEC3G by an HIV-1 Vif-Cullin5-Elongin B-Elongin C complex is essential for Vif function. J Biol Chem 280(19): 1857318578.

8. Mariani R, Chen D, Schrofelbauer B, Navarro F, Konig R, et al. (2003) Species-specific exclusion of APOBEC3G from HIV-1 virions by Vif. Cell 114(1): 21-31.

9. Mehle A, Goncalves J, Santa Marta M, Mc Pike M, Gabuzda D (2004) Phosphorylation of a novel SOCS-box regulates assembly of the HIV-1 Vif-Cul5 complex that promotes APOBEC3G degradation. Genes Dev 18(23): 2861-2866.

10. Mehle A, Strack B, Ancuta P, Zhang C, Mc Pike M, et al. (2004) Vif overcomes the innate antiviral activity of APOBEC3G by promoting its degradation in the ubiquitin-proteasome pathway. J Biol Chem 279(9): 7792-7798.

11. Stopak K, De Noronha C, Yonemoto W, Greene WC (2003) HIV-1 Vif blocks the antiviral activity of APOBEC3G by impairing both its translation and intracellular stability. Mol Cell 12(3): 591-601.

12. Wichroski MJ, Ichiyama K, Rana TM (2005) Analysis of HIV-1 viral infectivity factor-mediated proteasome-dependent depletion of APOBEC3G: correlating function and subcellular localization. J Biol Chem 280(9): 8387-8396.

13. Yu X, Yu Y, Liu B, Luo K, Kong W, et al. (2003) Induction of APOBEC3G ubiquitination and degradation by an HIV-1 Vif-Cul5-SCF complex. Science 302(5647): 1056-1060.

14. Letko M, Booiman T, Kootstra N, Simon V, Ooms M (2015) Identification of the HIV-1 Vif and Human APOBEC3G Protein Interface. Cell Reports 13(9): 1789-1799.

15. Marassi FM, Ma C, Gratkowski H, Straus SK, Strebel K, et al. (1999) Correlation of the structural and functional domains in the membrane protein Vpu from HIV-1. Proc Natl Acad Sci USA 96(25): 14336-1434.
16. Schubert U, Bour S, Ferrer Montiel AV, Montal M, Maldarelli F, et al. (1996) The two biological activities of human immunodeficiency virus type $1 \mathrm{Vpu}$ protein involve two separable structural domains. J Virol 70(2): 809-819.

17. Willey RL, Maldarelli F, Martin MA, Strebel K (1992) Human immunodeficiency virus type 1 Vpu protein induces rapid degradation of CD4. J Virol 66(12): 7193-7200.

18. Balliet JW, Kolson DL, Eiger G, Kim FM, Mc Gann KA, et al. (1994) Distinct effects in primary macrophages and lymphocytes of the human immunodeficiency virus type 1 accessory genes vpr, vpu, and nef : Mutational analysis of a primary HIV-1 isolate. Virology 200(2): 623631.

19. Geraghty RJ, Talbot KJ, Callahan M, Harper W, Panganiban AT (1994) Cell type-dependence for Vpu function. J Med Primatol 23(2-3): 146-150.

20. Sakai H, Tokunaga K, Kawamura M, Adachi A (1995) Function of human immunodeficiency virus type 1 Vpu protein in various cell types. J Gen Virol 76(Pt 11): 2717-2722.

21. Ewart GD, Sutherland T, Gage PW, Cox GB (1996) The Vpu protein of human immunodeficiency virus type 1 forms cation-selective ion channels. J Virol 70(10): 7108-7115.

22. Deora A, Ratner L (2001) Viral protein U (Vpu)-mediated enhancement of human immunodeficiency virus type 1 particle release depends on the rate of cellular proliferation. J Virol 75(14): 6714-6718.

23. Neil SJ, Sandrin V, Sundquist WI, Bieniasz PD (2007) An interferonalpha-induced tethering mechanism inhibits HIV-1 and Ebola virus particle release but is counteracted by the HIV-1 Vpu protein. Cell Host Microbe 2(3): 193-203.

24. Neil SJ, Zang T, Bieniasz PD (2008) Tetherin inhibits retrovirus release and is antagonized by HIV-1 Vpu. Nature 451(7177): 425-430.

25. Kupzig S, Korolchuk V, Rollason R, Sugden A, Wilde A, et al. (2003) Bst-2/ HM1.24 is a raft-associated apical membrane protein with an unusual topology. Traffic 4(10): 694-709.

26. Sauter D, Vogl M, Kirchhoff F (2011) Ancient origin of a deletion in human BST2/tetherin that confers protection against viral zoonoses. Hum Mutat 32(11): 1243-1245.

27. Sauter D, Schindler M, Specht A, Landford WN, Münch J, et al. (2009) Tetherin-driven adaptation of Vpu and Nef function and the evolution of pandemic and nonpandemic HIV-1 strains. Cell Host Microbe 6(5): 409-421.

28. Jia B, Serra Moreno R, Neidermyer W, Rahmberg A, Mackey J, et al. (2009) Species-specific activity of SIV Nef and HIV-1 Vpu in overcoming restriction by tetherin/BST2. PLoS Pathog 5(5): e1000429.

29.Zhang F, Wilson SJ, Landford WC, Virgen B, Gregory D, et al. (2009) Nef proteins from simian immunodeficiency viruses are tetherin antagonists. Cell Host Microbe 6(1): 54-67.

30. Sauter D, Unterweger D, Vogl M, Usmani SM, Heigele A, et al. (2012) Human tetherin exerts strong selection pressure on the HIV-1 group $\mathrm{N}$ Vpu protein. PLoS Pathog 8(12): e1003093.

31. Yang SJ, Lopez LA, Hauser H, Exline CM, Haworth KG, et al. (2010) Antitetherin activities in Vpu- expressing primate lentiviruses. Retrovirology $7: 13$.

32. Inuzuka M, Hayakawa M, Ingi T (2005) Serinc, an activity-regulated protein family, incorporates serine into membrane lipid synthesis. The Journal of Biological Chemistry 280(42): 35776-3578.

33. Usami Y, Wu Y, Göttlinger HG (2015) SERINC3 and SERINC5 restrict HIV1 infectivity and are counteracted by Nef. Nature 526(7572): 218-223.

34. Pereira EA, Da Silva LLP (2016) HIV-1 Nef: taking control of protein trafficking. Traffic 17(9):976-996.

35. Trautz B, Pierini V, Wombacher R, Stolp B, Chase AJ, et al. (2016) The antagonism of HIV-1 Nef to SERINC5 particle infectivity restriction 
involves the counteraction of virion-associated pools of the restriction factor. Journal of Virology 90(23): 10915-10927.

36. Aiken C, Konner J, Landau NR, Lenburg ME, Trono D (1994) Nef induces CD4 endocytosis: requirement for a critical dileucine motif in the membrane-proximal CD4 cytoplasmic domain. Cell 76(5): 853-864.

37. Seaton KE, Smith CD (2008) N-Myristoyltransferase isozymes exhibit differential specificity for human immunodeficiency virus type $1 \mathrm{Gag}$ and Nef. The Journal of General Virology 89(Pt 1): 288-296

38. Bentham M, Mazaleyrat S, Harris M (2006) Role of myristoylation and $\mathrm{N}$-terminal basic residues in membrane association of the human immunodeficiency virus type 1 Nef protein. Journal of General Virology 87(Pt 3): 563-571.

39. Cohen EA, Terwilliger EF, Jalinoos Y, Proulx J, Sodroski JG, et al. (1990) Identification of HIV-1 vpr product and function. J Acquir Immune Defic Syndr 3(1): 11-18.

40. Yuan X, Matsuda Z, Matsuda M, Essex M, Lee TH (1990) Human immunodeficiency virus vpr gene encodes a virion-associated protein. AIDS Res Hum Retroviruses 6(11): 1265-1271.

41. Lewis PF, Emerman M (1994) Passage through mitosis is required for oncoretroviruses but not for the human immunodeficiency virus. J Virol 68(1): 510-516.

42. Roe T, Reynolds TC, Yu G, Brown PO (1993) Integration of murine leukemia virus DNA depends on mitosis. Embo J 12(5): 2099-2108.

43. Desai TM, Marin M, Sood C, Shi J, Nawaz F, et al. (2015) Fluorescent protein-tagged Vpr dissociates from HIV-1 core after viral fusion and rapidly enters the cell nucleus. Retrovirology 12: 88

\section{ISSN: 2574-1241}

DOI: 10.26717/BJSTR.2020.31.005120

Lopez Hernandez MA. Biomed J Sci \& Tech Res

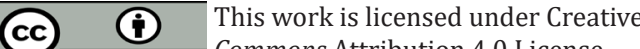

Commons Attribution 4.0 License

Submission Link: https://biomedres.us/submit-manuscript.php
44. He J, Choe S, Walker R, Di MP, Morgan DO, et al. (1995) Human immunodeficiency virus type 1 viral protein $\mathrm{R}(\mathrm{Vpr})$ arrests cells in the G2 phase of the cell cycle by inhibiting p34cdc2 activity. J Virol 69(11): 6705-6711.

45. Jowett JB, Planelles V, Poon B, Shah NP, Chen ML, et al. (1995) The human immunodeficiency virus type 1 vpr gene arrests infected T cells in the G2 + M phase of the cell cycle. J Virol 69(10): 6304-6313.

46. Re F, Braaten D, Franke EK, Luban J (1995) Human immunodeficiency virus type $1 \mathrm{Vpr}$ arrests the cell cycle in G2 by inhibiting the activation of p34cdc2-cyclin B. J Virol 69(11): 6859-6864.

47. Arunagiri C, Macreadie I, Hewish D, Azad A (1997) A C-terminal domain of HIV-1 accessory protein Vpr is involved in penetration, mitochondrial dysfunction and apoptosis of human CD4+ lymphocytes. Apoptosis 2(1): 69-76

48. Ayyavoo V, Mahboubi A, Mahalingam S, Ramalingam R, Kudchodkar S, et al. (1997) HIV-1 Vpr suppresses immune activation and apoptosis through regulation of nuclear factor $\kappa B$. Nat Med 3(10): 1117-1123.

49. Majumder B, Venkatachari NJ, Srinivasan A, Ayyavoo V (2009) HIV-1 mediated immune pathogenesis: Spotlight on the role of viral protein $\mathrm{R}$ (Vpr) Curr. HIV Res 7(2): 169-177.

50. Vermeire J, Roesch F, Sauter D, Rua R, Hotter D, et al. (2016) HIV triggers a cGAS-dependent, Vpu- and Vpr-regulated type I interferon response in CD4+ T cells. Cell Rep 17(2): 413-424.

51. Zhang S, Feng Y, Narayan O, Zhao LJ (2001) Cytoplasmic retention of HIV-1 regulatory protein Vpr by protein-protein interaction with a novel human cytoplasmic protein VprBP. Gene 263(1-2): 131-140.

$\begin{array}{ll}\text { BIOMEDICAL } & \text { Assets of Publishing with us } \\ \text { RESEARCHES } & \text { - Global archiving of articles } \\ \text { - Immediate, unrestricted online access } & \text { - Rigorous Peer Review Process } \\ & \text { - Authors Retain Copyrights } \\ \end{array}$

